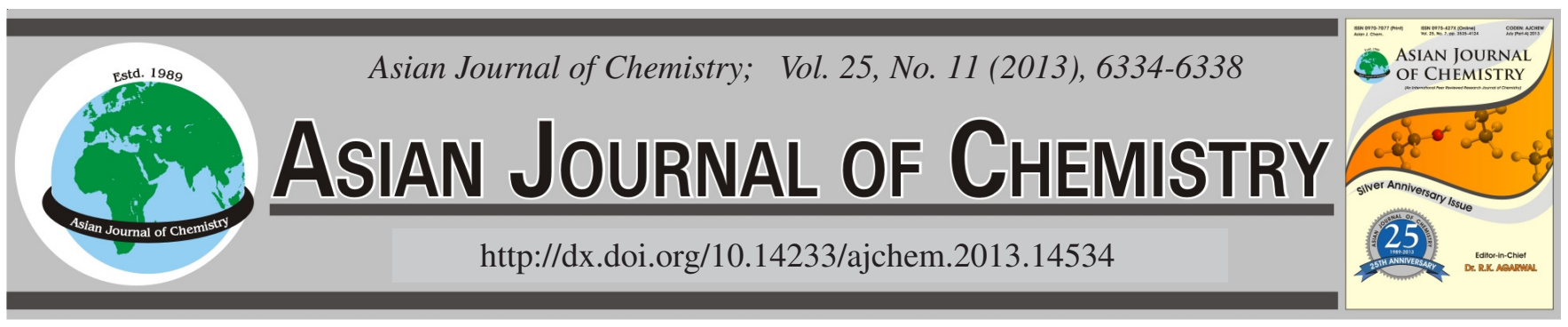

\title{
Synthesis of Potential Biologically Active 4,5-Dimethoxy-2- [3-oxo-3-phenylprop-1-en-1-yl]benzene Sulfonic Acid Derivatives
}

Bilal Shahid ${ }^{1, *}$, Muhammad Zia-Ur-Rehman ${ }^{2}$, Naveed Ahmed ${ }^{3}$, Muhammad Athar Abbasi $^{1}$ and Muhammad Zaheer ${ }^{2}$

${ }^{1}$ Department of Chemistry, Government College University Lahore-54000, Pakistan

${ }^{2}$ Applied Chemistry Research Centre, PCSIR Laboratories Complex Lahore-54600, Pakistan

${ }^{3}$ Forman Christian College (A Chartered University), Lahore-54600, Pakistan

*Corresponding author: E-mail: bilalshahid_1@hotmail.com; rehman_pcsir@hotmail.com

(Received: 6 September 2012;

Accepted: 8 May 2013)

AJC-13466

A series of potential biologically active 4,5-dimethoxy-2-[3-oxo-3-phenylprop-1-en-1-yl]benzenesulfonamides was synthesized. 3,4-
Dimethoxybenzaldehyde was condensed with acetophenone followed by its chlorosulfonation to get 4,5-dimethoxy-2-[3-oxo-3-phenylprop-1-
en-1-yl]benzenesulfonyl chloride, which was further reacted with a number of amines and hydrazides to get the 4,5-dimethoxy-2-[3-oxo-3-
phenylprop-1-en-1-yl]benzenesulfonic acid derivatives. All the synthesized compounds were subjected to preliminary evaluation for their anti-
bacterial activity against selected strains of gram positive and gram negative bacteria. Some of the assayed compounds showed marked activity.
Key Words: Chalcones, Sulfonamides, Antibacterial activity.

\section{INTRODUCTION}

The term "chalcone" is a general term used to describe compounds with the 1,3-diphenylprop-2-en-1-one (I) structure. A large number of 1,3-di-substituted prop-2-ene-1-one derivatives have been synthesized up till now. These compounds are precursors of flavonoids and iso-flavonoids, which are abundant in plant kingdom ${ }^{1}$. Basic structure of 1,3-di- substituted prop-2-ene-1-one nucleus is configured in Fig. 1 having two aromatic rings joined by a three carbon $\alpha-\beta$ unsaturated carbonyl systems.<smiles>O=C(C=Cc1ccccc1)c1ccccc1</smiles>

Fig. 1. An example of substituted prop-2-en-1-one

Chalcones naturally occur in different plant species like Angelica, Glycyrrhiza, Humulus and Scutellaria and constitute an important class of flavonoid family. These possess a wide spectrum of biological activities, including antibacterial, antifungal $^{3}$, antiinflammatory ${ }^{4}$, antitumor ${ }^{5}$ and antimutagenic ${ }^{6}$. Such compounds have also been found active for the inhibition of chemical mediator's release ${ }^{7}$, leukotriene $\mathrm{B} 4^{8}$, nitric oxide ${ }^{9}$, tyrosinases ${ }^{10}$ and aldose reductase activities ${ }^{11}$. These have also the ability to inhibit the cancer cell proliferation ${ }^{12}$ in vivo and show a significant activity against cancer ${ }^{13,14}$. Induction of apoptosis in many cell types specially the breast cancers is one of the major properties of chalcones ${ }^{15}$ (Fig. 2).

Similarly, sulfonamides are much familiar for their versatile nature of biological activities. These are found to possess anti $\mathrm{HIV}^{16}$, antiinflammatory ${ }^{17}$ and antimicrobial activities $^{18}$ (Fig. 3). Chalcones with sulfonylamino side chain derivatives are very important category of $\alpha$-glycosidase inhibitors ${ }^{19}$. In the past, most of the antimalarial drugs were based on sulfonamide derivatives and were effective against Plasmodium falciparum ${ }^{20}$.<smiles>CC(C)=CCOc1ccc(/C=C/C(=O)c2ccc(OCC=C(C)C)cc2OCC(=O)O)cc1</smiles><smiles>CCOc1cc(O)c(CC=C(C)C)c(O)c1C(=O)/C=C/c1ccc(O)cc1</smiles>

$\mathrm{H}_{3} \mathrm{C}^{-}$

Xanthohumol - An anticancer prenylflavonoid MCHP - An insulin mimetic chalcone Fig. 2. Structures of few medicinally important 1,3-di-substituted prop-2ene-1-ones 

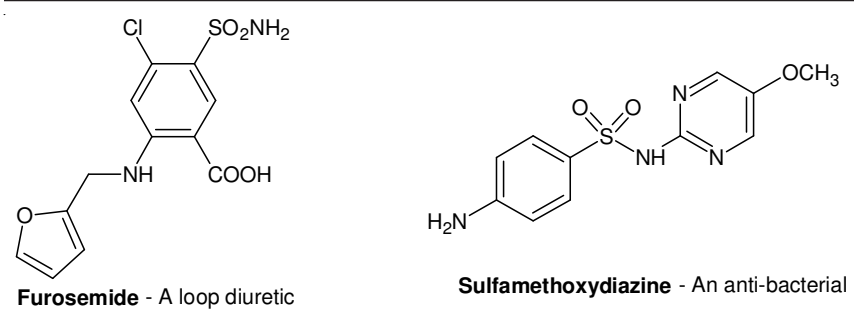<smiles>Cc1ccccc1N1C(=O)c2cc(S(N)(=O)=O)c(Cl)cc2NC1C</smiles><smiles>COc1ccc(NS(=O)(=O)c2ccc(N)cc2)nn1</smiles>

Fig. 3. Structures of some known sulfonamide drugs

Keeping in view the potential biological activities possessed by different compounds having the basic structure of 1,3disubstituted prop-2-en-1-one (Fig. 1) and sulfonamides. It was perceived that synergism of both the moieties into a single nucleus, may give rise to more potent compounds. In this quest, novel 4,5-dimethoxy-2-[3-oxo-3-phenylprop-1-en-1-yl]benzenesulfonamides (6a-n) were synthesized from 3,4dimethoxychalcone and subjected to antibacterial activity (Scheme-I).

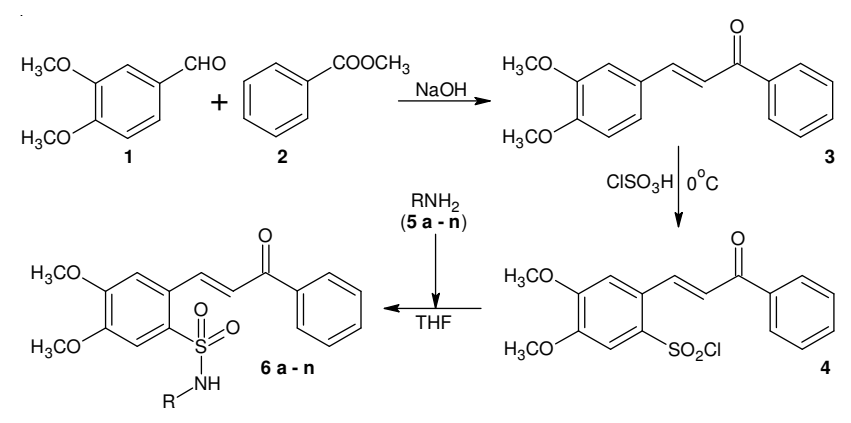

Scheme-I: Schematic diagram of the reactions involved

\section{EXPERIMENTAL}

Melting points were taken on Gallenkemp melting point apparatus and are uncorrected. ${ }^{1} \mathrm{H}$ NMR spectra were taken on the Bruker DPX-400 NMR spectrometer and chemical shifts are given in ppm downfield from TMS as the internal standard. IR spectra were recorded in the spectral range of 4000-400 $\mathrm{cm}^{-1}$ on Perkin Elmer 1600-FT spectrometer. Mass spectra were taken on Jeol SX-102 spectrometer. All the chemicals were purchased from E. Merck, BDH or Fluka and used without purification. However, solvents were purified through distillation.

3-(3,4-Dimethoxy-phenyl)-1-propenone (3): The product was synthesized according to the literature procedure ${ }^{21}$. A mixture of acetophenone (2) (12 g, $100 \mathrm{mmol}), 3,4$-dimethoxy benzaldehyde (1) (16.6 g, $100 \mathrm{mmol})$, sodium hydroxide $(11.84 \mathrm{~g})$, water $(106 \mathrm{~mL})$ and ethyl alcohol $(95 \% ; 66.8 \mathrm{~mL})$ was heated and stirred magnetically at room temperature for $3 \mathrm{~h}$. After completion of the reaction, the product was extracted in cold ethanol $(3 \times 25 \mathrm{~mL})$. The contents were dried to give crude product which was crystallized from methanol. Bright yellow crystalline solid; m.p. $75-78^{\circ} \mathrm{C}$. IR $\left(\mathrm{KBr}, v_{\max }, \mathrm{cm}^{-1}\right)$ : 1662, 1630, 1105. $\delta: 3.82$ (s, 3H, $\left.\mathrm{OCH}_{3}\right), 3.89$ (s, 3H, $\left.\mathrm{OCH}_{3}\right)$, 7.36-7.89 (m, 10H); MS m/z: $268[\mathrm{M}+1]$.

4,5-Dimethoxy-2-[3-oxo-3-phenylprop-1-en-1-yl]benzenesulfonyl chloride (4): To a stirred solution of chlorosulfonic acid (34.5 g, $0.3 \mathrm{~mol})$ at $0{ }^{\circ} \mathrm{C}, 3-(3,4-$ dimethoxyphenyl)-1propenone (3) (10 g, $0.038 \mathrm{~mol})$ was added. The dark red solution obtained by mixing was allowed to stir at room temperature for $3 \mathrm{~h}$. After completion of reaction, the contents were poured over crushed ice and the resulting precipitate was filtered, washed with water and dried. Recrystallization of the product from acetonitrile yielded compound 4 (11.1 g, 80.5 $\%$ ): m.p. $173-174{ }^{\circ} \mathrm{C}$. IR (KBr, $\left.v_{\max }, \mathrm{cm}^{-1}\right): 1326,1655,1126$. ${ }^{1} \mathrm{H}$ NMR (DMSO- $\left.d_{6}\right) \delta: 3.82\left(\mathrm{~s}, 3 \mathrm{H}, \mathrm{OCH}_{3}\right), 3.89\left(\mathrm{~s}, 3 \mathrm{H}, \mathrm{OCH}_{3}\right)$, 7.36-7.89 (m. 9H); MS m/z: $366\left[\mathrm{M}^{+}\right], 368\left[\mathrm{M}^{+}+2\right]$.

General procedure for the synthesis of 4,5-dimethoxy2-(3-oxo-3-phenylprop-1-en-1-yl)benzenesulfonamides (6a-n): A mixture of 4,5-dimethoxy-2-[3-oxo-3-phenylprop1-en-1-yl]benzenesulfonyl chloride (4) (3.67 g, $100 \mathrm{mmol}$ ) and appropriate amine/hydrazine/ hydrazide (5a-n) (100 $\mathrm{mmol})$ in tetrahydrofuran $(100 \mathrm{~mL})$ was heated on water bath at $80^{\circ} \mathrm{C}$ (for reaction time; Table-1). After completion of the reaction, contents were poured over ice cooled water and the products were extracted with dichloromethane. Solvent was evaporated under reduced pressure to get the crude product which was recrystallized from methanol and dried to give crystalline products.

4,5-Dimethoxy- $N$-(4-nitrophenyl)-2-(3-oxo-3-phenylprop-1-en-1-yl)benzenesulfonamide (6a): Blood red crystals, m.p. $204-206^{\circ} \mathrm{C}$. IR $\left(\mathrm{KBr}, v_{\max }, \mathrm{cm}^{-1}\right): 1384,1635,3543 .{ }^{1} \mathrm{H}$ NMR (DMSO-d $\left.d_{6}\right) \delta: 3.78\left(\mathrm{~s}, 3 \mathrm{H}, \mathrm{OCH}_{3}\right), 3.87\left(\mathrm{~s}, 3 \mathrm{H}, \mathrm{OCH}_{3}\right)$, 5.23 (s, 1H, NH), 7.31 (s, 1H, ArH), 7.43 (s, 1H, ArH), 7.46 $(\mathrm{d}, 1 \mathrm{H}, J=15.6, \mathrm{C}=\mathrm{CH}), 7.56-7.62(\mathrm{~m}, 4 \mathrm{H}, \mathrm{ArH}), 7.82-7.93$ (m, 5H, ArH), 8.78 (d, 1H, $J=15.6, \mathrm{HC}=\mathrm{C}) . \mathrm{MS} \mathrm{m} / \mathrm{z}: 468$ $\left[\mathrm{M}^{+}\right]$. Anal. calcd. (\%) for $\mathrm{C}_{23} \mathrm{H}_{20} \mathrm{~N}_{2} \mathrm{O}_{7} \mathrm{~S}: \mathrm{C}, 58.97 ; \mathrm{H}, 4.30 ; \mathrm{N}$, 5.98; Found (\%): C, 58.94; H, 4.32; N, 5.99.

TABLE-1

REACTION OF 4,5-DIMETHOXY-2-[3-OXO-3-PHENYLPROP-1-EN-1-YL] BENZENESULFONYL CHLORIDE (4) WITH AMINO COMPOUNDS (5a-n)

\begin{tabular}{|c|c|c|c|c|c|c|c|}
\hline Entry & Product & $\begin{array}{l}\text { Reaction time } \\
\text { (h) }\end{array}$ & Yield (\%) & Entry & Product & $\begin{array}{l}\text { Reaction time } \\
\text { (h) }\end{array}$ & Yield (\%) \\
\hline 1 & $6 \mathbf{a}$ & 4 & 84 & 8 & $6 h$ & 7 & 78 \\
\hline 2 & $6 b$ & 3 & 72 & 9 & $6 \mathbf{i}$ & 5 & 85 \\
\hline 3 & $6 c$ & 4 & 89 & 10 & $6 \mathbf{j}$ & 4 & 88 \\
\hline 4 & 6d & 6 & 82 & 11 & $6 \mathbf{k}$ & 5 & 80 \\
\hline 5 & $6 e$ & 6 & 81 & 12 & 61 & 4 & 92 \\
\hline 6 & $6 f$ & 4 & 80 & 13 & $6 \mathrm{~m}$ & 5 & 90 \\
\hline 7 & $6 \mathrm{~g}$ & 7 & 80 & 14 & 6n & 6 & 84 \\
\hline
\end{tabular}


$N$-(2-Aminoethyl)-4,5-dimethoxy-2-(3-oxo-3-phenylprop-1-en-1-yl)benzenesulfonamide (6b): Off-white crystalline solid, m.p. $209-211^{\circ} \mathrm{C}$. IR ( $\left.\mathrm{KBr}, \mathrm{v}_{\max }, \mathrm{cm}^{-1}\right)$ : 1408.56 , 1624.20, 3551.13. ${ }^{1} \mathrm{H}$ NMR (DMSO- $\left.d_{6}\right) \delta: 3.75\left(\mathrm{~s}, 3 \mathrm{H}, \mathrm{OCH}_{3}\right)$, 3.86 (s, $\left.3 \mathrm{H}, \mathrm{OCH}_{3}\right), 4.01$ (s, $\left.2 \mathrm{H}, \mathrm{NH}_{2}\right), 4.73-4.79$ (m, $\left.2 \mathrm{H}, \mathrm{CH}_{2}\right)$, $5.30(\mathrm{~s}, 1 \mathrm{H}, \mathrm{NH}), 6.69-6.72\left(\mathrm{~m}, 2 \mathrm{H}, \mathrm{CH}_{2}\right), 7.37$ (s, 1H, ArH), 7.45 (s, 1H, ArH), 7.49 (d, 1H, $J=15.6, \mathrm{C}=\mathrm{CH}), 7.66-7.69$ $(\mathrm{m}, 5 \mathrm{H}, \mathrm{ArH}), 8.80(\mathrm{~d}, 1 \mathrm{H}, J=15.6, \mathrm{HC}=\mathrm{C}) \mathrm{MS}$ $\mathrm{m} / \mathrm{z}: 390\left[\mathrm{M}^{+}\right]$. Anal. calcd. (\%) for $\mathrm{C}_{19} \mathrm{H}_{22} \mathrm{~N}_{2} \mathrm{O}_{5} \mathrm{~S}: \mathrm{C}, 58.45 ; \mathrm{H}$, 5.68; N, 7.17; Found (\%): C, 58.48; H, 5.66; N, 7.20.

2-[4,5-Dimethoxy-2-(3-oxo-3-phenylprop-1-en-1-yl)phenylsulfonamido]benzoic acid (6c): Light Green crystals; m.p. $190{ }^{\circ} \mathrm{C}$. IR (KBr, $\left.v_{\max }, \mathrm{cm}^{-1}\right): 1420,1637,3547,3595 .{ }^{1} \mathrm{H}$ NMR (DMSO) $\delta: 3.77\left(\mathrm{~s}, 3 \mathrm{H}, \mathrm{OCH}_{3}\right), 3.89\left(\mathrm{~s}, 3 \mathrm{H}, \mathrm{OCH}_{3}\right)$, $5.30(\mathrm{~s}, 1 \mathrm{H}, \mathrm{NH}), 7.44$ (s, 1H, ArH), $7.50(\mathrm{~s}, 1 \mathrm{H}, \mathrm{ArH}), 7.55$ (t, $1 \mathrm{H}, J=7.6, \mathrm{ArH}), 7.58(\mathrm{~d}, J=15.6,1 \mathrm{H}, \mathrm{C}=\mathrm{CH}), 7.69-7.73$ (m, 4H, ArH), 8.10-8.17 (m, 4H, ArH), 8.77 (d, 1H, $J=15.6$, $\mathrm{HC}=\mathrm{C}) 12.43(\mathrm{~s}, 1 \mathrm{H}, \mathrm{OH}) . \mathrm{MS} \mathrm{m} / \mathrm{z}: 467\left[\mathrm{M}^{+}\right]$. Anal. calcd. (\%) for $\mathrm{C}_{24} \mathrm{H}_{21} \mathrm{NO}_{7} \mathrm{~S}: \mathrm{C}, 61.66 ; \mathrm{H}, 4.53 ; \mathrm{N}, 3.0$; found (\%): C, $61.63 ; \mathrm{H}, 4.59 ; \mathrm{N}, 3.09$

3-[4,5-Dimethoxy-2-(3-oxo-3-phenylprop-1-en-1-yl)phenylsulfonamido]benzoic acid (6d): Dark grey crystals, m.p. $201-203{ }^{\circ} \mathrm{C}$. IR (KBr, $\left.v_{\max }, \mathrm{cm}^{-1}\right)$ : 1442, 1622, 3509, 3572. ${ }^{1} \mathrm{H}$ NMR (DMSO- $\left.d_{6}\right) \delta: 3.78\left(\mathrm{~s}, 3 \mathrm{H}, \mathrm{OCH}_{3}\right), 3.87(\mathrm{~s}, 3 \mathrm{H}$, $\left.\mathrm{OCH}_{3}\right), 5.34$ (s, 1H, NH), 7.43 (s, 1H, ArH), 7.49 (s, 1H, ArH), 7.54 (s, 1H, ArH), 7.57 (d, $J=15.6,1 \mathrm{H}, \mathrm{C}=\mathrm{CH}), 7.67-7.74$ (m, 5H, ArH), 8.12-8.18 (m, 3H, ArH), 8.78 (d, 1H, $J=15.6$, $\mathrm{HC}=\mathrm{C}) 12.47(\mathrm{~s}, 1 \mathrm{H}, \mathrm{OH}) . \mathrm{MS} \mathrm{m} / \mathrm{z}: 467\left[\mathrm{M}^{+}\right]$. Anal. calcd. (\%) for $\mathrm{C}_{24} \mathrm{H}_{21} \mathrm{NO}_{7} \mathrm{~S}: \mathrm{C}, 61.66 ; \mathrm{H}, 4.53 ; \mathrm{N}, 3.0$; found (\%): C, $61.60 ; \mathrm{H}, 4.56 ; \mathrm{N}, 3.09$

2-Chloro-5-(4,5-dimethoxy-2-(3-oxo-3-phenylprop-1en-1-yl)phenylsulfonamido)benzoic acid (6e): Light brown solid, m.p. $210^{\circ} \mathrm{C}$. IR (KBr, $\left.v_{\max }, \mathrm{cm}^{-1}\right)$ : 1420.56, 1672.38, 3570.29, 3568.10, 683.56. ${ }^{1} \mathrm{H}$ NMR (DMSO- $\left.d_{6}\right) \delta: 3.78(\mathrm{~s}$, $\left.3 \mathrm{H}, \mathrm{OCH}_{3}\right), 3.87\left(\mathrm{~s}, 3 \mathrm{H}, \mathrm{OCH}_{3}\right), 5.34(\mathrm{~s}, 1 \mathrm{H}, \mathrm{NH}), 7.43(\mathrm{~s}, 1 \mathrm{H}$, ArH), 7.49 (s, 1H, ArH), 7.55 (s, 1H, ArH), 7.59 (d, $J=15.6$, $1 \mathrm{H}, \mathrm{C}=\mathrm{CH}), 7.67-7.74(\mathrm{~m}, 5 \mathrm{H}, \mathrm{ArH}), 8.07-8.11(\mathrm{~m}, 2 \mathrm{H}, \mathrm{ArH})$, $8.72(\mathrm{~d}, 1 \mathrm{H}, J=15.6, \mathrm{HC}=\mathrm{C}), 12.49$ (s, 1H, OH). MS m/z: $501\left[\mathrm{M}^{+}\right], 503\left[\mathrm{M}^{+}+2\right]$. Anal. calcd. $(\%)$ for $\mathrm{C}_{24} \mathrm{H}_{20} \mathrm{NO}_{7} \mathrm{SCl}: \mathrm{C}$, 57.43; H, 4.02; N, 2.79; found (\%): C, 57.42; H,3.97; N, 2.81.

$\mathrm{N}$-Hydroxy-4,5-dimethoxy-2-(3-oxo-3-phenylprop-1en-1-yl)benzenesulfonamide (6f): Pale yellow solid, m.p. 162 ${ }^{\circ} \mathrm{C}$. IR (KBr, $\left.v_{\max }, \mathrm{cm}^{-1}\right): 1384.08,1635.99,3550.29 .{ }^{1} \mathrm{H}$ NMR $\left(\mathrm{DMSO}-d_{6}\right) \delta: 3.78\left(\mathrm{~s}, 3 \mathrm{H}, \mathrm{OCH}_{3}\right), 3.83\left(\mathrm{~s}, 3 \mathrm{H}, \mathrm{OCH}_{3}\right), 5.31$ (s, 1H, NH), 7.39 (s, 1H, ArH), 7.47 (s, 1H, ArH), 7.48 (d, 1H, $J=15.6, \mathrm{C}=\mathrm{CH}), 7.65-7.67(\mathrm{~m}, 5 \mathrm{H}, \mathrm{ArH}), 8.83(\mathrm{~d}, 1 \mathrm{H}, J=$ 15.6, $\mathrm{HC}=\mathrm{C}), 13.01(\mathrm{~s}, 1 \mathrm{H}, \mathrm{OH})$. MS m/z: $363\left[\mathrm{M}^{+}\right]$. Anal. calcd. $(\%)$ for $\mathrm{C}_{17} \mathrm{H}_{17} \mathrm{NO}_{6} \mathrm{~S}$ : C, 56.19; $\mathrm{H}, 4.72 ; \mathrm{N}, 3.85$; found $(\%)$ : C, 56.22; H, 4.69; N, 3.86

4,5-Dimethoxy-2-(3-0xo-3-phenylprop-1-en-1-yl)- $N^{\prime}$ phenylbenzenesulfonohydrazide $(6 \mathrm{~g})$ : Black crystalline solid, m.p. $190-193^{\circ} \mathrm{C}$. IR ( $\left.\mathrm{KBr}, \nu_{\max }, \mathrm{cm}^{-1}\right)$ : $1383.89,1635.53$, 3544.98. ${ }^{1} \mathrm{H}$ NMR (DMSO- $d_{6}$ ) $\delta: 3.78$ (s, $\left.3 \mathrm{H}, \mathrm{OCH}_{3}\right), 3.88$ (s, $\left.3 \mathrm{H}, \mathrm{OCH}_{3}\right), 5.33(\mathrm{~s}, 1 \mathrm{H}, \mathrm{NH}), 7.45(\mathrm{~s}, 1 \mathrm{H}, \mathrm{ArH}), 7.48(\mathrm{~d}, 1 \mathrm{H}$, $J=15.6, \mathrm{C}=\mathrm{CH}), 7.51(\mathrm{~s}, 1 \mathrm{H}, \mathrm{ArH}), 8.80(\mathrm{~d}, 1 \mathrm{H}, J=15.6$, $\mathrm{HC}=\mathrm{C}$ ), 7.59-7.64 (m, 5H, ArH), 8.11-8.17(m, 5H, ArH), 9.32 (s, $1 \mathrm{H}, \mathrm{NH}) . \mathrm{MS} \mathrm{m} / \mathrm{z}: 438\left[\mathrm{M}^{+}\right]$. Anal. calcd. (\%) for $\mathrm{C}_{23} \mathrm{H}_{22} \mathrm{~N}_{2} \mathrm{O}_{5} \mathrm{~S}$ : C, 63.0; H, 5.06; N, 6.39; found (\%): C, 63.03; $\mathrm{H}, 5.04 ; \mathrm{N}, 6.36$.
$N^{\prime}$-(3,5-Dinitrophenyl)-4,5-dimethoxy-2-(3-oxo-3phenylprop-1-en-1-yl)benzenesulfonohydrazide (6h): Light red solid, m.p. $275^{\circ} \mathrm{C}$. IR (KBr, $\left.v_{\max }, \mathrm{cm}^{-1}\right): 1449.54,1653.71$, 1105.28, 3544.98. ${ }^{1} \mathrm{H}$ NMR (DMSO- $\left.d_{6}\right) \delta: 3.77$ (s, $3 \mathrm{H}, \mathrm{OCH}_{3}$ ), 3.89 (s, 3H, $\left.\mathrm{OCH}_{3}\right), 5.34$ (s, 1H, NH), 7.41 (s, 1H, ArH), 7.48 (s, 1H, ArH), 7.51(d, 1H, J = 15.6, C=CH), $7.56(\mathrm{~s}, 1 \mathrm{H}, \mathrm{ArH})$, $8.80(\mathrm{~d}, 1 \mathrm{H}, J=15.6, \mathrm{HC}=\mathrm{C}), 7.59-7.64(\mathrm{~m}, 2 \mathrm{H}, \mathrm{ArH}), 8.11-$ 8.17 (m, 5H, ArH), 9.32(s, 1H, NH). MS m/z: 528 [M+ $\mathrm{M}^{+}$. Anal. calcd. (\%) for $\mathrm{C}_{23} \mathrm{H}_{20} \mathrm{~N}_{4} \mathrm{O}_{9} \mathrm{~S}: \mathrm{C}, 52.27 ; \mathrm{H}, 3.81 ; \mathrm{N}, 10.60$; found (\%): C, 52.24; H, 3.82; N, 10.66.

2-[(4,5-Dimethoxy-2-(3-oxo-3-phenylprop-1-en-1yl)phenyl)sulfonyl]hydrazinecarboxamide (6i): Orange crystalline solid, m.p. $197^{\circ} \mathrm{C}$. IR (KBr, $\left.v_{\max }, \mathrm{cm}^{-1}\right)$ : 1408.83 , 1661.24, 3540. ${ }^{1} \mathrm{H}$ NMR (DMSO-d $\left.d_{6}\right) \delta: 3.78$ (s, 3H, $\mathrm{OCH}_{3}$ ), $3.85\left(\mathrm{~s}, 3 \mathrm{H}, \mathrm{OCH}_{3}\right), 4.01\left(\mathrm{~s}, 2 \mathrm{H}, \mathrm{NH}_{2}\right), 5.31(\mathrm{~s}, 1 \mathrm{H}, \mathrm{NH}), 7.39$ (s, 1H, ArH), 7.47 (s, 1H, ArH), 7.48(d, 1H, J=15.6, C=CH), 7.65-7.67 (m, 5H, ArH), 8.83 (d, 1H, $J=15.6, \mathrm{HC}=\mathrm{C}), 9.34$ (s, $1 \mathrm{H}, \mathrm{NH}) . \mathrm{MS} \mathrm{m} / \mathrm{z}: 405\left[\mathrm{M}^{+}\right]$. Anal. calcd. (\%) for $\mathrm{C}_{18} \mathrm{H}_{19} \mathrm{~N}_{3} \mathrm{O}_{6} \mathrm{~S}$ : C, 53.32; H, 4.72; N, 10.36; found (\%): C, 53.35; $\mathrm{H}, 4.71 ; \mathrm{N}, 10.34$.

4,5-Dimethoxy- $N$ '-benzoyl-2-(3-oxo-3-phenylprop-1en-1-yl)benzenesulfonohydrazide (6j): Light brown crystalline solid, m.p. $220^{\circ} \mathrm{C}$. IR ( $\left.\mathrm{KBr}, v_{\max }, \mathrm{cm}^{-1}\right): 1419.42,1651.52$, 3531.89. ${ }^{1} \mathrm{H}$ NMR (DMSO- $\left.d_{6}\right) \delta: 3.79\left(\mathrm{~s}, 3 \mathrm{H}, \mathrm{OCH}_{3}\right), 3.88(\mathrm{~s}$, $\left.3 \mathrm{H}, \mathrm{OCH}_{3}\right), 5.33(\mathrm{~s}, 1 \mathrm{H}, \mathrm{NH}), 7.43(\mathrm{~s}, 1 \mathrm{H}, \mathrm{ArH}), 7.49(\mathrm{~s}, 1 \mathrm{H}$, $\mathrm{ArH}), 8.80(\mathrm{~d}, 1 \mathrm{H}, J=15.6, \mathrm{HC}=\mathrm{C}), 7.55(\mathrm{~m}, 1 \mathrm{H}, \mathrm{C}=\mathrm{CH})$, 8.10 (d, 2H, $J=7.2$, ArH), 7.94 (d, 2H, $J=7.2$, ArH), 7.267.62 (m, 6H, ArH), 9.30 (s, 1H, NH). MS m/z: 466 [M+'. Anal. calcd. (\%) for $\mathrm{C}_{24} \mathrm{H}_{22} \mathrm{~N}_{2} \mathrm{O}_{6} \mathrm{~S}$ : C, 61.79; H, 4.75; N, 6.0; found (\%): C, 61.80; H, 4.72; N, 5.95.

4,5-Dimethoxy- $N^{\prime}$-(4-nitrobenzoyl)-2-(3-oxo-3-phenylprop-1-en-1-yl)benzenesulfonohydrazide (6k): Red crystalline solid, m.p. $193^{\circ} \mathrm{C}$. IR $\left(\mathrm{KBr}, v_{\max }, \mathrm{cm}^{-1}\right)$ : 1419.42, 1651.52, 3531.89. ${ }^{1} \mathrm{H}$ NMR (DMSO- $d_{6}$ ) $\delta: 3.78$ (s, $\left.3 \mathrm{H}, \mathrm{OCH}_{3}\right), 3.88$ (s, $\left.3 \mathrm{H}, \mathrm{OCH}_{3}\right), 5.33$ (s, 1H, NH), 7.45 (s, 1H, ArH), 7.48 (d, 1H, $J=15.6, \mathrm{C}=\mathrm{CH}), 7.50(\mathrm{~s}, 1 \mathrm{H}, \mathrm{ArH}), 8.80(\mathrm{~d}, 1 \mathrm{H}, J=15.6$, $\mathrm{HC}=\mathrm{C}), 7.58-7.63$ (m, 4H, ArH), 8.10-8.16 (m, 5H, ArH), 9.32 (s, $1 \mathrm{H}, \mathrm{NH}) . \mathrm{MS} \mathrm{m} / \mathrm{z}: 511\left[\mathrm{M}^{+}\right]$. Anal. calcd. (\%) for $\mathrm{C}_{24} \mathrm{H}_{21} \mathrm{~N}_{3} \mathrm{O}_{8} \mathrm{~S}$ : C, 56.35; H, 4.14; N, 8.22; found (\%): C, 56.33; $\mathrm{H}, 4.10 ; \mathrm{N}, 8.23$.

$N^{\prime}$-(2-Hydroxybenzoyl)-4,5-dimethoxy-2-(3-oxo-3phenylprop-1-en-1-yl)benzenesulfonohydrazide (61): White solid, m.p. $238{ }^{\circ} \mathrm{C}$. IR ( $\left.\mathrm{KBr}, v_{\max }, \mathrm{cm}^{-1}\right)$ : 1441.40, 1652.08, $3567.88,3505.32 .{ }^{1} \mathrm{H}$ NMR (DMSO- $\left.d_{6}\right) \delta: 3.83\left(\mathrm{~s}, 3 \mathrm{H}, \mathrm{OCH}_{3}\right)$, $3.87\left(\mathrm{~s}, 3 \mathrm{H}, \mathrm{OCH}_{3}\right), 5.36(\mathrm{~s}, 1 \mathrm{H}, \mathrm{NH}), 6.90(\mathrm{~d}, 2 \mathrm{H}, J=7.6$, ArH), 6.93 (d, 2H, J = 8.4, ArH), 7.32 (s, 1H, ArH), 7.46 (s, $1 \mathrm{H}, \mathrm{ArH}), 7.48(\mathrm{~d}, 1 \mathrm{H}, J=15.6, \mathrm{C}=\mathrm{CH}), 7.51$ (s, 1H, $\mathrm{ArH})$, 7.57-7.64 (m, 4H, ArH), $8.81(\mathrm{~d}, 1 \mathrm{H}, J=15.6, \mathrm{HC}=\mathrm{C}), 9.32$ (s, 1H, NH), 13.03 (s, 1H, OH ). MS m/z: $482\left[\mathrm{M}^{+}\right]$. Anal. calcd. (\%) for $\mathrm{C}_{24} \mathrm{H}_{22} \mathrm{~N}_{2} \mathrm{O}_{7} \mathrm{~S}: \mathrm{C}, 59.74 ; \mathrm{H}, 4.60 ; \mathrm{N}, 5.81$; found (\%): C, 59.71; H, 4.61; N, 5.83.

$N^{\prime}$-(4-Hydroxybenzoyl)-4,5-dimethoxy-2-(3-oxo-3phenylprop-1-en-yl)benzenesulfonohydrazide (6m): Grey crystalline solid, m.p. $215^{\circ} \mathrm{C}$. IR (KBr, $\left.v_{\max }, \mathrm{cm}^{-1}\right)$ : 1436.64 , 1623.26, 3534.05, 3503.81. ${ }^{1} \mathrm{H}$ NMR (DMSO- $d_{6}$ ) $\delta: 3.79$ (s, $\left.3 \mathrm{H}, \mathrm{OCH}_{3}\right), 3.86\left(\mathrm{~s}, 3 \mathrm{H}, \mathrm{OCH}_{3}\right), 5.35(\mathrm{~s}, 1 \mathrm{H}, \mathrm{NH}), 6.90$ (d, $2 \mathrm{H}, J=7.6, \mathrm{ArH}), 6.93$ (d, 2H, $J=8.4, \mathrm{ArH}), 7.32$ (s, 1H, ArH), 7.46 (s, 1H, ArH), $7.48(\mathrm{~d}, 1 \mathrm{H}, J=15.6, \mathrm{C}=\mathrm{CH}), 7.51$ (s, 1H, ArH), 7.53-7.64 (m, 4H, ArH), 8.81 (d, 1H, $J=15.6$, 
$\mathrm{HC}=\mathrm{C}), 9.31(\mathrm{~s}, 1 \mathrm{H}, \mathrm{NH}), 13.03(\mathrm{~s}, 1 \mathrm{H}, \mathrm{OH}) . \mathrm{MS} \mathrm{m} / \mathrm{z}: 482$

$\left[\mathrm{M}^{+}\right]$. Anal. calcd. $(\%)$ for $\mathrm{C}_{24} \mathrm{H}_{22} \mathrm{~N}_{2} \mathrm{O}_{7} \mathrm{~S}: \mathrm{C}, 59.74 ; \mathrm{H}, 4.60 ; \mathrm{N}$,

5.81; found (\%): C, 59.76; H, 4.59; N, 5.78 .

$N^{\prime}$-(4-Chlorobenzoyl)-4,5-dimethoxy-2-(3-oxo-3-phenylprop-1-en-1-yl)benzenesulfonohydrazide (6n): Pale yellow crystalline solid, m.p. $207^{\circ} \mathrm{C}$. IR $\left(\mathrm{KBr}, \mathrm{v}_{\max }, \mathrm{cm}^{-1}\right)$ : 1383.65 , 1660.56, 3503.66, 620.03. ${ }^{1} \mathrm{H}$ NMR (DMSO- $\left.d_{6}\right) \delta: 3.76(\mathrm{~s}$, $\left.3 \mathrm{H}, \mathrm{OCH}_{3}\right), 3.87\left(\mathrm{~s}, 3 \mathrm{H}, \mathrm{OCH}_{3}\right), 5.36(\mathrm{~s}, 1 \mathrm{H}, \mathrm{NH}), 7.45(\mathrm{~s}, 1 \mathrm{H}$, $\mathrm{ArH}), 7.48$ (d, 1H, $J=15.6, \mathrm{C}=\mathrm{CH}), 7.52$ (s, 1H, ArH), 7.597.64 (m, 3H, ArH), 8.07-8.13 (m, 6H, ArH), 8.81 (d, 1H, $J=$ 15.6, $\mathrm{HC}=\mathrm{C}), 9.31(\mathrm{~s}, 1 \mathrm{H}, \mathrm{NH}) . \mathrm{MS} \mathrm{m} / \mathrm{z}: 500\left[\mathrm{M}^{+}\right]$. Anal. calcd. (\%) for $\mathrm{C}_{24} \mathrm{H}_{21} \mathrm{~N}_{2} \mathrm{O}_{6} \mathrm{SCl}$ : C, 57.54; H, 4.23; N, 5.59; found (\%): C, 57.57; H, 4.21; N, 5.68.

Antibacterial activity: All the newly synthesized compounds (dissolved in dimethylformamide) were subjected to antimicrobial screening by determining the minimum inhibitory concentration (MIC) using the agar dilution technique ${ }^{22}$. The in vitro antimicrobial activity of the prepared compounds (6a-n) against three gram negative bacteria i.e., Escherichia coli, Pseudomonas aeruginosa, Salmonella typhi and four gram positive bacteria i.e., Bacillus cereus, Bacillus subtilis, Bacillus thuringiensis and Staphylococcus aureus was determined by preparing suspensions of each micro-organism to contain approximately $10^{5}-10^{6} \mathrm{CFU}$ (colony forming units)/ well. The test compounds were applied to the wells at concentrations ranging from 200 to $3.0 \mathrm{mg} \mathrm{mL}^{-1}$ in DMF solution, in addition to the 0 (control) and the standard, streptomycin. The plates were incubated for $24 \mathrm{~h}$ at $37^{\circ} \mathrm{C}$ and growth assessed by visual inspection. The minimum inhibitory concentration (MIC) was defined as the lowest concentration of inhibitor at which microbial growth was not apparent disregarding a single colony or a faint haze caused by the inoculums.

\section{RESULTS AND DISCUSSION}

3-(3,4-Dimethoxyphenyl)-1-propenone (3) synthesized by usual Claisen Schmidt condensation was chlorosulfonylated using chlorosulphonic acid at $0{ }^{\circ} \mathrm{C}$ to yield 4,5-dimethoxy-2[3-oxo-3-phenylprop-1-en-1-yl]benzenesulfonyl chloride (4). It was found that reaction temperature plays crucial role for the reaction. Slow addition of the chalcone to chlorosulfonic acid at $0{ }^{\circ} \mathrm{C}$ yields a single desired product, while addition at slight higher temperatures ended up with unextractable multispotted product containing dichlorosulfonylated products. This sulfonyl chloride (4) was then reacted with a number of amino compounds (amines, hydrazines and hydrazides) to get the corresponding sulphonamides (6a-f) and sulfonohydrazides (6g-n) (Table-2). All of the newly synthesized compounds were characterized through spectroscopic techniques (FT-IR, ${ }^{1} \mathrm{H}$
NMR and mass spectrometry) along with their elemental analyses and were found in accordance with the calculated values.

All the newly synthesized compounds (dissolved in dimethylformamide) were subjected to antibacterial screening against four gram positive bacteria (Bacillus cereus, Bacillus subtilis, Bacillus thuringiensis and Staphylococcus aureus) and three gram negative bacteria (Escherichia coli, Pseudomonas aeruginosa, Salmonella typhi) by determining their minimum inhibitory concentrations (MICs) using agar dilution technique $^{23}$ and streptomycin as reference drug. The choice of streptomycin as a clinical standard was based on the fact that at low concentrations, it only inhibits growth of the bacteria through induction of prokaryotic ribosomes to misread mRNA. Streptomycin also prevents initiation of protein synthesis and leads to death of microbial cells. Also, in humans, they have structurally different ribosomes from bacteria, thereby allowing the selectivity of this antibiotic for bacteria ${ }^{24}$.

The MICs of the active compounds against the susceptible pathogenic organisms are presented in Table-3. The results showed that in general, compounds (6a-f) synthesized by the reaction of sulfonyl chloride (4) with amines were found active against gram positive bacteria while sulfonohydrazides (6gn) were found more active against gram negative bacteria. An insight to the activities of different compounds obtained reveals that the compounds (6a-n) are more active against Bacillus species (B. subtilis, B. cereus, B. thuringiensis) than that of $S$. aureus. It is also evident that the compounds $(\mathbf{6 a}, \mathbf{6} \mathbf{b}$ and $\mathbf{6 f})$ are more active than the others perhaps due to the presence of nitrogen atoms with lone pairs. In contrast to the behaviour shown by the compounds (6a-f), sulfonohydrazides (6g-n) showed their activity against gram negative bacteria. Compounds bearing nitro, hydroxyl and chloro groups were found active and their activity is in the following order: $\mathrm{NO}_{2}>p-\mathrm{OH}>$ $p-\mathrm{Cl}>o-\mathrm{OH}$. Rest of the compounds were either found inactive or with MIC greater than 15 .

\section{Conclusion}

The present study revealed that synergism of sulfonamide and sulfonohydrazide moieties to 3-(3,4-dimethoxyphenyl)1-propenone could be useful as a template for future development through modification or derivatization to design a more potent biologically active compounds. The new skeleton may also possess other biological activities of the parent ring systems.

\section{ACKNOWLEDGEMENTS}

The authors are grateful to Pakistan Council of Scientific \& Industrial Research Laboratories Complex, Lahore for the provision of bench facilities and chemicals.

TABLE-2

REACTANT AMINES USED FOR THE SYNTHESIS OF TITLE COMPOUNDS (5a-n)

\begin{tabular}{cl|ll}
\hline Reactant amines & \multicolumn{1}{|c}{ Name } & Reactant amines & \multicolumn{1}{c}{ Name } \\
\hline $\mathbf{5 a}$ & 4-Nitroaniline & $\mathbf{5 h}$ & Dinitrophenyl hydrazine \\
$\mathbf{5 b}$ & Ethane-1,2-dimaine & $\mathbf{5 i}$ & Semicarbazide hydrochloride \\
$\mathbf{5 c}$ & 2-Aminobenzoic acid & $\mathbf{5 j}$ & Benzohydrazide \\
$\mathbf{5 d}$ & 3-Aminobenzoic acid & $\mathbf{5 k}$ & 4-Nitrobenzohydrazide \\
$\mathbf{5 e}$ & 5-Amino-2-chlorobenzoic acid & $\mathbf{5 l}$ & 2-Hydroxybenzohydrazide \\
$\mathbf{5 f}$ & Hydroxylamine hydrochloride & $\mathbf{5 m}$ & 4-Hydroxybenzohydrazide \\
$\mathbf{5 g}$ & Phenylammoniumchloride & $\mathbf{5 n}$ & 4-Chlorobenzohydrazide \\
\hline
\end{tabular}




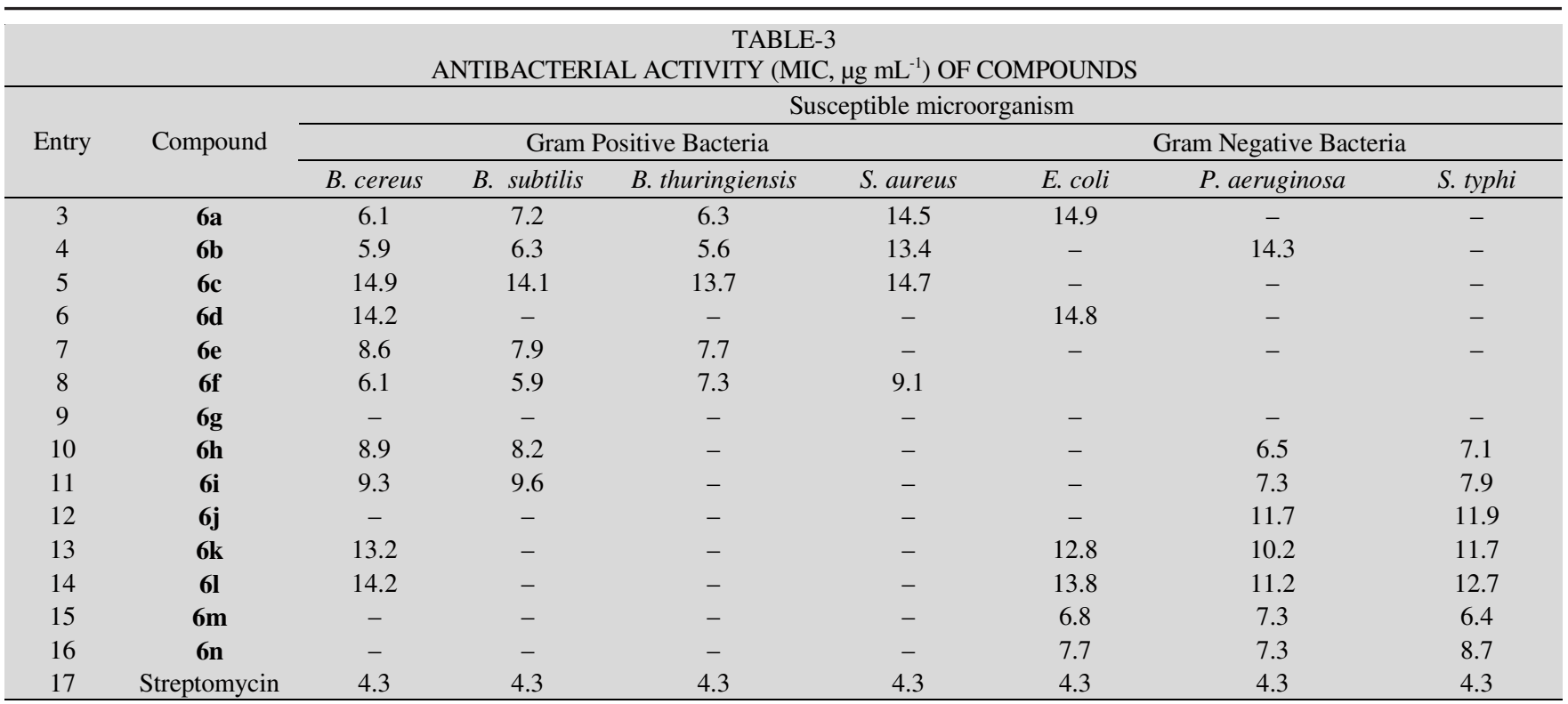

\section{REFERENCES}

1. N. Shimada, T. Aoki, S. Sato, Y. Nakamura, S. Tabata and S. Ayabe, Plant Physiol., 131, 941 (2003).

2. T. Hussain, H.L. Siddiqui, M. Zia-ur-Rehman, M.M. Yasinzai and M. Parvez, Eur. J. Med. Chem., 44, 4654 (2009).

3. S. Ducki, IDrugs, 10, 42 (2007).

4. N.J. Lawrence and A. T. McGown, Curr. Pharm. Des., 11, 1679 (2005).

5. Z. Nowakowska, Eur. J. Med. Chem., 42, 125 (2007).

6. J.R. Dimmock, D.W. Elias, M.A. Beazely and N.M. Kandepu, Curr. Med. Chem., 6, 1125 (1999).

7. H.H. Ko, L.T. Tsao, K.L. Yu, C.T. Liu, J.P. Wang and C.N. Lin, Bioorg. Med. Chem., 11, 105 (2003).

8. A.M. Deshpande, N.P. Argade, A.A. Natu and J. Eckman, Bioorg. Med. Chem., 7, 1237 (1999)

9. J. Rajas, J.M. Paya, J.N. Dominguez and M.L. Ferrandiz, Bioorg. Med. Chem. Lett., 12, 1951 (2002)

10. S. Khatib, O. Nerya, R. Musa, M. Shmnel, S. Tamir and J. Vaya, Bioorg. Med. Chem., 13, 433 (2005).

11. F. Severi, S. Benvenuti, L. Costantino, G. Vampa, M. Melegari and L. Antolini, Eur. J. Med. Chem., 33, 859 (1998)

12. S. Padhye, A. Ahmad, N. Oswal and F. H Sarkar, J. Hematol. Oncol., 2, 38 (2009).

13. Y. Satomi, Int. J. Cancer, 55, 506 (1993).
14. S. Yamamoto, E. Aizu, H. Jiang, T. Nakadate, I. Kiyoto, J.C.Wang and R. Kato, Carcinogenesis, 12, 317 (1991).

15. C.A. Calliste, J.C. Le Bail, P. Trouillas, C. Pouget, G. Habrioux, A.J. Chulia and J.L. Duroux, Anti-cancer Res., 21, 3949 (2001).

16. M. Liu, P. Wilairat, S.L. Croft, A. Lay-Choo Tan and M.-L. Go, Bioorg. Med. Chem., 11, 2729 (2003).

17. J.N. Dominguez, J.E. Charis, G. Lobo, N.G. de Domínguez, M.M. Moreno, F. Riggione, E. Sanchez, J. Olson and P.J. Rosenthal, Eur. J. Med. Chem., 36, 555 (2001).

18. N. Ahmed, M. Zia-ur-Rehman, H.L. Siddiqui, M.F. Ullah and M. Parvez, Eur. J. Med. Chem., 46, 2368 (2009).

19. W.D. Seo, J.H. Kim, J.E. Kang, H.W. Ryu, M.J.C. Long, H.S. Lee, M.S. Yang and K.H. Park, Bioorg. Med. Chem. Lett., 15, 5514 (2005).

20. J.N. Domínguez, C. León, J. Rodrigues, N.G. de Domínguez, J. Gut and P. J. Rosenthal, Farmaco, 60, 307 (2005).

21. V. Shettigar, J. B.-J. Teh, H.-K. Fun, I. A. Razak, P. S. Patil and S. M. Dharmaprakash, Acta Cryst., E62, o4646 (2006).

22. J.G. Colle, J.P. Duguid, A.G. Fraser and B.P. Marmion, in eds.: T.J. Mackie and J.E. McCartney, Practical Medical Biology, Churchill Livingstone, London, UK, Vol. 13 (1989).

23. H. Hanel and W. Raether, Mycoses, 31, 148 (1988).

24. C. Booth, in eds.: J.R. Norris and D.W. Ribbons, Methods in Microbiology, Academic Press, London, New York, Vol. IV, p. $49-94$ (1977). 